\title{
Network reorganization driven by temporal interdependence of its elements
}

\author{
Jack Waddell \\ Department of Physics, University of Michigan, Ann Arbor, Michigan 48109 \\ Michal Zochowski \\ Department of Physics, Biophysics Research Division, University of Michigan, Ann Arbor, Michigan 48109
}

(Received 1 November 2005; accepted 6 March 2006; published online 11 May 2006)

\begin{abstract}
We employ an adaptive parameter control technique based on detection of phase/lag synchrony between the elements of the system to dynamically modify the structure of a network of nonidentical, coupled Rössler oscillators. Two processes are simulated: adaptation, under which the initially different properties of the units converge, and rewiring, in which clusters of interconnected elements are formed based on the temporal correlations. We show how those processes lead to different network structures and investigate their optimal characteristics from the point of view of resulting network properties. (C) 2006 American Institute of Physics. [DOI: 10.1063/1.2189972]
\end{abstract}

We applied two control schemes based on the detection of temporal interdependencies on a network of chaotic oscillators and evaluate the self-organization on the network structure. The first scheme is the parameter adaptive control to which we refer to as adaptation. Adaptation acts through the regularity in relative time lag (lag synchrony) between pairs of coupled oscillators to alter their initially different frequencies in order to obtain their convergence. The second scheme, which we refer to as rewiring, modifies the network structure (topology of connections as well as their relative strength). Rewiring strengthens or weakens coupling between any pair of oscillators depending on the asymmetrical properties of lag synchrony modeled roughly after biological processes of long term potentiation and depression of the synapses. We find that the relative speeds of the two control processes lead to different final networks, ranging in coupling strength and density of clustering, and show the existence of a set of relative control speeds that optimize coupling stability. Also, using an asymmetric form of rewiring where strengthening and weakening rates are different, we show that a set of asymmetric rates optimize the density of clustering while retaining other network features, such as cluster size and selectivity.

\section{INTRODUCTION}

Recently a lot of research has been focused on the role of network structure in network function and the existence of the feedback between the two. The network topologies were studied extensively in disease spread, ${ }^{1,2}$ authors' citations, 3,4 neural networks, ${ }^{5}$ and other types of social, biological, and artificial interactions. Other research has examined the dynamics of network characteristics ${ }^{6,7}$ and of dynamical systems acting over networks. ${ }^{8-11}$

In neuronal systems network plasticity - involving modulatory changes taking place on individual neurons, as well as those underlying modifications of network connectivity -is thought to underlie learning and memory formation. It has been shown experimentally that the firing patterns of individual neurons can be changed by the alteration of the ionic currents, a process that may occur depending on the behavioral state (i.e., network dynamics) of an animal. ${ }^{12}$ On the other hand, the synaptic coupling of some neuronal classes has been shown to increase or decrease depending on the temporal interdependencies of their relative firing patterns. Long term potentiation (LTP) strengthens synapses from the leading to the following neuron when the latter fires consistently within a short $(\approx 100 \mathrm{~ms})$ time window after the former. Long term depression (LTD) on the other hand decreases synaptic efficacy if the "follower" neuron fires before its supposed triggering neuron. ${ }^{13-15}$ Both types of neural plasticity, adaptation of individual cells or ensembles and synaptic modifications, will in turn modify the network dynamics.

Here, we investigate how temporal pattern formation may interact with network topology. We study a generic network of coupled, nonidentical Rössler oscillators that incorporate two basic mechanisms of activity dependent plasticity: activity dependent adaptation (implemented as parameter adaptive control $^{16-18}$ ) where the internal dynamical characteristics of the coupled oscillators are modified by the properties of the temporal interactions with other units, and network rewiring mechanisms that are taken from neurophysiology and are simulating activity dependent synaptic modifications (ADSM). The evolving temporal patterning in the system is monitored through an adaptive measure that we have developed earlier ${ }^{19}$ that monitors temporal phase/lag interdependence between the pairs of the oscillators. We have already shown that such a measure provides means to obtain parameter adaptive control in the systems of two coupled, non-identical, non-linear oscillators. ${ }^{20}$

We show that the detection of the structure of temporal interdependencies can successfully drive network restructuring. In the case when only adaptation is present, the elements of the network adapt to the same value of their natural frequency forming a network of identical elements. We found that this adaptation is much faster and more robust with the networks having global connectivity as compared to the net- 
works with only local connections. On the other hand, the presence of only the rewiring mechanism promotes formation of small directional clusters with connections leading from elements of higher frequency to the elements with lower frequency. We have also found that asymmetry in the speed of rewiring of network connections leads to an optimal network structure, where the cluster formation is highly selective but the clusters remain dense. Lastly, interaction of adaptation and rewiring mechanisms leads to formation of clusters with feedback connections between adapted nodes. Here we show that the interplay of these two processes may have constructive as well as destructive results; for a range of the relative values of rates for the two processes the networks are effectively abolished, while other ranges result in networks with various structures.

\section{MODELED NETWORK}

The network consists of $N$ nonidentical Rössler oscillators described by the following equations:

$$
\begin{aligned}
& x_{i}=-\omega_{i} y_{i}-z_{i}, \\
& y_{i}=\omega_{i} x_{i}+a y_{i}+\sum_{j=1}^{N} \alpha_{i j}\left(y_{j}-y_{i}\right), \\
& z_{i}=b+\left(x_{i}-c\right) z_{i},
\end{aligned}
$$

where the parameters $(a, b, c)=(0.3,0.2,10)$ are the Rössler parameters, $\omega_{i}=[1,1.1] \omega_{0}$ is the frequency of oscillator $i$, and $\alpha_{i j}$ is the coupling strength from $j$ to $i$. The parameter $\omega_{0}=1$ sets the relative time scale of the dynamics. The initial coupling is assigned on a $2 \mathrm{D}$ toroidal lattice with a connectivity radius $r=1$ or $r=2$ and is drawn from a uniform, random distribution over $[0.3,0.5]$. The oscillators are initially integrated for 20000 steps (with a step size $\delta t=0.051 / \omega_{0}$ ) before either control process is initiated.

It has been established that synchrony in a system of coupled oscillators can be divided into three major classes: complete synchronization if the coupled units are identical and their trajectories are fully synchronized, ${ }^{21,22}$ generalized synchronization if there is a functional dependence between the temporal dynamics of the coupled units, ${ }^{23,24}$ and phase synchronization, appearing in the systems of weakly coupled nonidentical oscillators when the phases are locked but amplitudes of the trajectories are uncorrelated. ${ }^{25-27} \mathrm{Lag}$ synchrony is usually established as a transition from phase synchrony for stronger coupling regimes. ${ }^{28}$

\section{A. Adaptive measure of temporal ordering}

Causal entropy (CE) (Ref. 19) is an asymmetric, timeresolved measure of the regularity of time or phase lag between two series of discrete events. Here the timing of an event is defined as a time at which trajectory of the individual oscillator crosses Poincaré section $z=1$. For time lag measurement, the $\mathrm{CE}_{i j}$ is calculated from a histogram of $\Delta t_{i j}$, a series of interevent intervals between an event generated by the $i$ th oscillator and the preceding event on the $j$ th oscillator [see Fig. 1(A)]. Conversely, $\mathrm{CE}_{j i}$ is based on the distribution

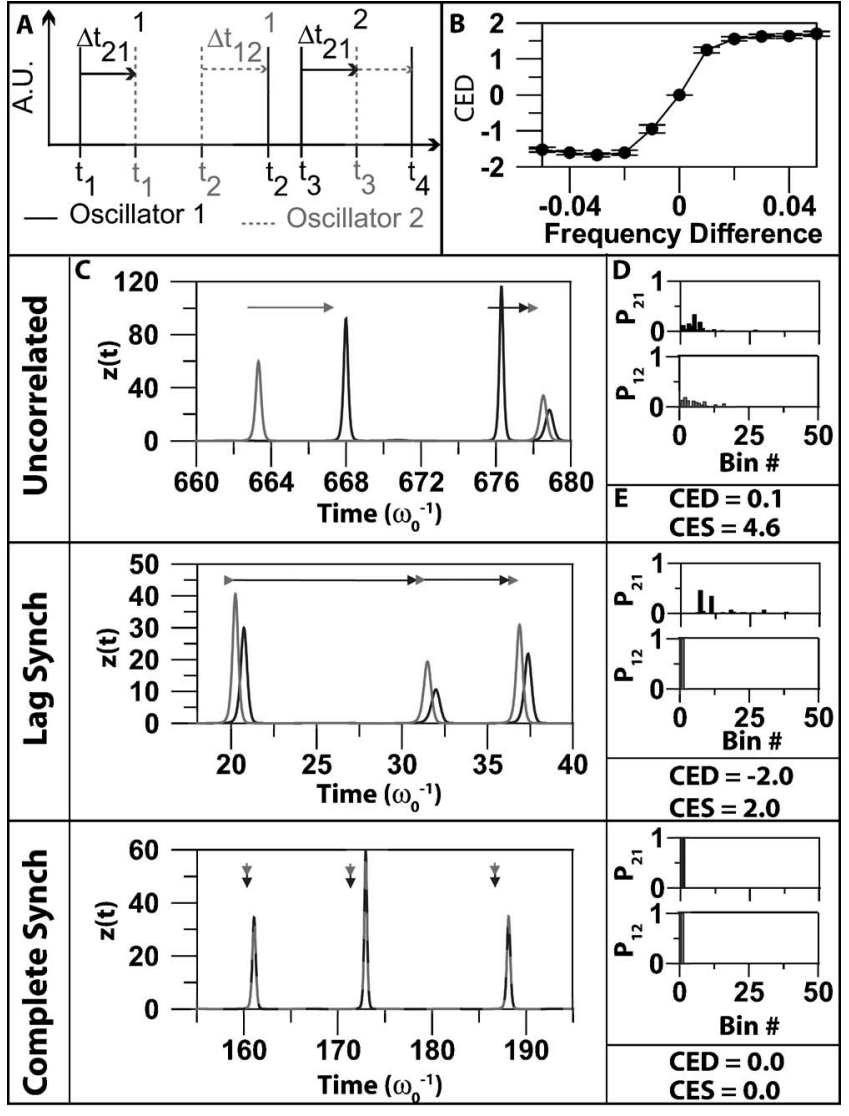

FIG. 1. (A) The example of time intervals used to form the histogram $P_{i j}$. Only the interval from the most recent event of $j$ is used for $\Delta t_{i j}$. (B) The difference of causal entropy, $\left(\mathrm{CED}=\mathrm{CE}_{12}-\mathrm{CE}_{21}\right)$, between two mutually coupled Rössler oscillators with differing frequencies. Oscillator 1 has $\omega$ $=1.05$, and the frequency of oscillator 2 is varied between $\omega \in[1.00,1.10]$. The causal entropy successfully identifies the temporal ordering between the pair. (C-E) Examples of three pairs of Rössler oscillators in different dynamical regimes. Each shows: a close-up segment of $z(t)$ for both oscillators, with arrows indicating the interevent intervals used in the CE calculation (i); a pair of histograms of $P$ developed from the intervals (ii), generated at the end of the integration; the causal entropy difference (CED) and sum (CES) calculated from the histograms (iii). (C) Example of a pair of uncorrelated (uncoupled) oscillators, both with $\omega=1$. The interevent intervals are irregular for both oscillators (panels $\mathrm{Ci}$ and $\mathrm{Cii}$ ). This results in a small CED, but a large CES (Ciii). (D) Example of a coupled pair of lagsynchronized oscillators, with different frequencies $\left(\alpha_{12}=\alpha_{21}=0.5, \omega_{1}\right.$ $=1, \omega_{2}=1.2$ ). Oscillator 1 follows 2 regularly (panels Di and Dii), while 2 does not follow 1 with a fixed pattern. This results in a large CED and a CES of similar magnitude to the CED (panel Diii). (E) Example of a pair of completely synchronized oscillators, with $\alpha_{12}=\alpha_{21}=0.5, \omega_{1}=\omega_{2}=1.0$. Both oscillators fire simultaneously (panel Ei and Eii; note that in Ei, the gray line depicting trajectory of oscillator 2 is dashed such that the trajectory of oscillator 1 may be viewed beneath). This leads to both a small CED and a small CES (panel Eiii).

of interevent durations calculated between an event that was generated by the $j$ th oscillator and the preceding event generated by the $i$ th oscillator.

Specifically, after each event $a$ of $i, \Delta t_{i j}^{a}$ is obtained, the corresponding bin of distribution $P_{i j}$ is updated by adding a value $\Delta P$, after which the entire histogram is normalized by $(1+\Delta P)$. Thus $P_{i j}$ is the estimated probability density of the interevent intervals of the $i$ th oscillator following the $j$ th. The normalization attenuates bins that have not been updated over the course of $n$ events by $(1+\Delta P)^{-n}$. This allows the 
measure to automatically adapt to the statistics of most recent interevent intervals. The Shannon entropy is then calculated

$$
\mathrm{CE}_{i j}(t)=-\sum_{k} P_{i j k} \times \log \left(P_{i j k}\right)
$$

where $k$ indexes the bins of the histogram. Since $\mathrm{CE}_{i j}$ $\neq \mathrm{CE}_{j i}$, the CE's provide asymmetric measurement of temporal interdependence of the events generated by both oscillators. A low $\mathrm{CE}_{i j}$ indicates that an event on the $i$ th oscillator appears systematically after an event on the $j$ th oscillator with a highly regular time lag, whereas a high value of $\mathrm{CE}_{i j}$ indicates large variability in the event pattern on the oscillator $i$ with respect to the events on oscillator $j$, indicating lack of temporal interlocking between the two oscillators. Explicitly, the possible situations that can be detected by the $\mathrm{CE}$ measurement are:

- $\mathrm{CE}_{i j} \approx \mathrm{CE}_{j i} \gg 0$. This indicates that the interevent distributions of both oscillators are fairly wide and thus the trajectories of the oscillators do not reveal any temporal interdependencies, implying lack of direct or indirect coupling [see Fig. 1(C)].

- $\mathrm{CE}_{i j} \approx 0$ and $\mathrm{CE}_{j i} \gg 0$. This implies that the $i j$ th distribution is narrow whereas the jith distribution is wide, indicating persistent temporal lag of the trajectory of the $i$ th oscillator relative to the trajectory of the $j$ th oscillator [see Fig. 1(D)].

- $\mathrm{CE}_{i j} \gg 0$ and $\mathrm{CE}_{j i} \approx 0$. This implies the reverse situation persistent temporal lag of the trajectory of the $j$ th oscillator relative to the trajectory of the $i$ th oscillator.

- $\mathrm{CE}_{i j} \approx \mathrm{CE}_{j i} \approx 0$. Both distributions are peaked. This usually implies complete synchronization of both oscillators [see Fig. 1(E)]. The last part of this condition, both entropies equal to zero, is relaxed in the presence of noise (see Ref. 19). The other possibility is that both processes are periodic and then the temporal interdependence cannot be determined solely based on lag variability.

To detect asymmetry in the temporal interdependencies between neural activities we monitor the causal entropy difference, $\mathrm{CED}_{i j}=\mathrm{CE}_{i j}-\mathrm{CE}_{j i}$. This measures the temporal ordering of the pair of oscillators [Fig. 1(B)].

\section{B. Modulation by adaptation and rewiring based on temporal patterning in the network}

We model two types of network structure modifications occurring based on the temporal interdependence between the oscillators: adaptation, which is implemented as parameter adaptive control and modulates the frequency of the oscillators, and rewiring, which controls formation (and/or strengthening) and abolition (and/or weakening) of the coupling between the oscillators.

Adaptation is governed by

$$
\begin{aligned}
& \Delta \omega_{i}=\sum_{j=1, k_{i, \mathrm{in}}} \frac{\beta_{\omega}}{k_{i, \mathrm{in}}}\left(\mathrm{CE}_{j i}-\mathrm{CE}_{i j}\right), \\
& \omega_{i}(t+d t)=\omega_{i}(t)+\Delta \omega_{i},
\end{aligned}
$$

with $\omega_{i}$ constrained to the range $[1,1.1] ; k_{i, \text { in }}$ is the number of connections into the $i$ th oscillator; the sum occurs over oscillators directly coupled to the $i$ th oscillator. The adaptation speed parameter, $\beta_{\omega}$, governs the rate at which adaptation occurs. In an illustrative example, consider two oscillators $i$ and $j$ that are mutually coupled to only one another, having frequencies such that $\omega_{i}>\omega_{j}$. In this case, $i$ will tend to fire shortly before $j$, leading to a low $\mathrm{CE}_{j i}$ and a high $\mathrm{CE}_{j i}$. Adaptation will cause $\omega_{i}$ to decrease and $\omega_{j}$ to increase until such time as the two completely synchronize, which occurs only when the frequencies become essentially identical.

Rewiring is governed by a rule strengthening couplings for which strong lag synchronization occurs and weakening them otherwise. It is given by the following:

$$
\begin{aligned}
& \Delta \alpha_{i j}=\frac{\beta_{\alpha}}{N}\left(\mathrm{CE}_{\text {threshold }}-\mathrm{CE}_{i j}\right), \\
& \alpha_{i j}(t+d t)=\alpha_{i j}(t)+\Delta \alpha_{i j},
\end{aligned}
$$

where $\beta_{\alpha}$ is the rewiring speed parameter, $\mathrm{CE}_{\text {threshold }}=0.5$ is threshold value at which the switch from coupling strengthening to weakening takes place and $N$ is the number of oscillators in the network. The coupling is constrained so that $\alpha_{i j} \in[0,0.5]$. If a coupling converges to zero, the coupling is destroyed. Conversely, if coupling does not exist, it is created when $\Delta \alpha$ is positive. The rewiring term is evaluated between all pairs of oscillators, regardless of the current existence of coupling. Studies of similar Hebbian rules on identical chaotic oscillators result in a transformation from regular to small world networks. ${ }^{29}$

\section{MEASUREMENT OF INTERACTION BETWEEN NETWORK TOPOLOGY AND DYNAMICS}

The simulations are divided into three groups. In the first set of simulations we measure properties of adaptation of individual neurons as a function of network connectivity and network topology. In the second set we measure effects of temporal patterning on network rewiring. Finally, in the third set of experiments we combine the two mechanisms together.

To determine the progress of adaptation in the network we measure either the time it takes for adaptation to complete or the resulting variance of the adapted oscillator frequencies after a given period of simulation time.

To measure the extent of adaptation for the combined case, where both the adaptation and network rewiring is present, we calculate local variance of oscillator frequency amongst members of formed clusters.

To characterize the network topology we measure the maximum size of the clusters, and the average weighted transitivity amongst the oscillators. Transitivity, also known as the clustering coefficient, is defined as the number of subclusters formed from triangles of coupled oscillators in a network divided by the number of possible oscillator pairs 
within this cluster (with proper constants such that the maximum is 1 for a completely connected cluster). Thus it can be understood as the probability, given that two oscillators both have couplings to a shared third oscillator, that there is a coupling between them. The transitivity, averaged over the whole network, measures the tendency for clusters to exist and to be densely intraconnected.

We modified the standard definition of clustering coefficient for weighted connection to be

$$
\operatorname{WCC} 1(\nu)=\sum_{i, j \in G_{1}(\nu)} \frac{\alpha_{i j}}{\alpha_{\max } k(k-1)},
$$

where the sum over the coupling strengths $\alpha_{i j}$ takes into account each directed coupling only once in the 1-neighborhood of oscillator $\nu, \alpha_{\max }$ is the maximum allowed coupling strength, and $k$ is the total number of couplings of the oscillator.

To detect global ordering in the system we monitor the direction of the phase shifts of the oscillators. It is known that the oscillators having higher internal frequency will lead those with lower frequency [see Fig. 1(B)]. We define an expectivity function which measures temporal ordering in the network with respect to frequencies of the individual oscillators

$$
E=\frac{1}{N(N-1)} \sum_{i, j, i \neq j}^{N} w_{i j},
$$

where

$$
w_{i j}= \begin{cases}1 & \text { if }\left(\mathrm{CE}_{j i}-\mathrm{CE}_{i j}\right)\left(\omega_{j}-\omega_{i}\right)>0 \\ -1 & \text { if }\left(\mathrm{CE}_{j i}-\mathrm{CE}_{i j}\right)\left(\omega_{j}-\omega_{i}\right) \leqslant 0\end{cases}
$$

Thus the expectivity function measures whether the predictions of time ordering of the oscillators based on the relative frequency values are in agreement with those calculated using CEs. If the proper value from a given pair is predicted correctly the function is assigned the value $w_{i j}=1$, and conversely if the prediction fails $w_{i j}=-1$.

\section{A. Adaptation of the individual oscillators in the network}

Here the network is allowed to adjust the frequencies of individual oscillators [Eqs. (2)], but network rewiring does not take place. An example of the behavior of such network can be seen in Fig. 2. The initial frequencies of individual oscillators were generated at random. The oscillators are represented by circles with different shades of gray, with white and black denoting lowest and highest frequency respectively [Fig. 2(A)]. During the evolution of the system the frequencies of the oscillator converge to single value making all oscillators identical and completely synchronized [Fig. 2(B)].

We examined the relationship between the degree of connectivity in the network and the speed of adaptation convergence (Fig. 3). The convergence is achieved even for very low connectivity $(\approx 5 \%)$, and the convergence time decreases with the increased connectivity. This is due to the fact that the temporal ordering in the system increases with

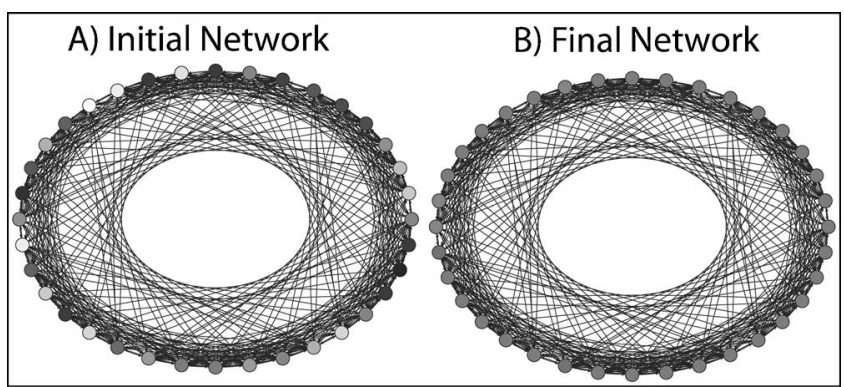

FIG. 2. A network undergoing adaptation $\left(N=36, r=2, \beta_{\omega}=0.0001\right)$. The darkness of the oscillators denotes the frequency, with white representing 1.0 and black 1.1. By the end of the run time of $20000 \omega_{0}^{-1}$ the entire network is highly adapted, with all nodes having virtually the same frequency.

the number of connections in the network; the expectivity function [Eq. (5)] increases with the connectivity value, indicating an increased degree of temporal ordering in the system during initial stages of the simulation.

We have also studied the adaptability of the network as a function of network topology. The ability for the network to adapt was compared over network topologies that range from locally connected regular networks to those with random global couplings. We have used the Watts-Strogatz (WS) Small World Network framework to achieve the intermediate network topologies. Specifically, the network of $(N=36)$ oscillators had initially only local connections formed within a radius $r=2$. Every coupling was then randomly rewired to form random connections with WS rewiring parameter $P$ (Fig. 4).

The convergence of adaptation time decreases as a function of the WS rewiring parameter $P$, indicating that networks having global connectivity adapt faster. This is again due to the fact that temporal ordering in the network depends on the network topology; the expectivity function increases significantly with the WS rewiring parameter $P$. This in turn is due to the reduction of average characteristic length in the

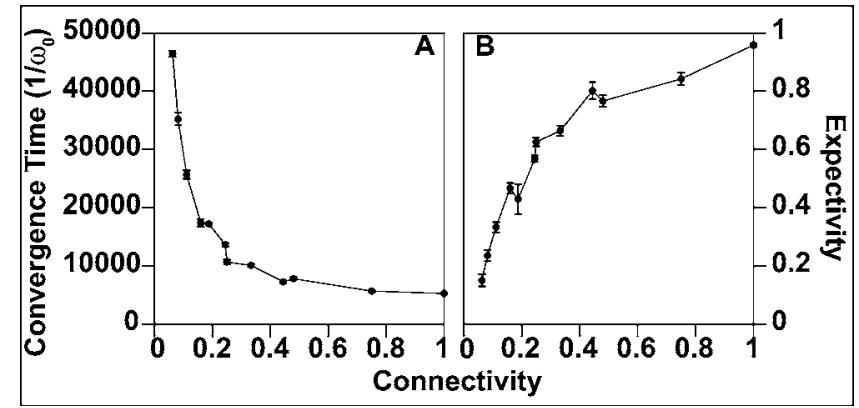

FIG. 3. Networks with $N=\{9,16,25,36,49,64\}$ and $r=\{1,2\}$ were generated and allowed to adapt with speed $\beta_{\omega}=0.0001$, after and an initial free period of 20000 iterations $\left(1000 \omega_{0}^{-1}\right)$. (A) Convergence time of adaptation vs network connectivity. The time required to completely adapt the network (when the rate of change in standard deviation of frequencies was less than $2 \%$ of the initial value) was recorded. More connected networks, which have small average distance between oscillators, complete adaptation more quickly than less connected networks. (B) Expectivity vs network connectivity. The time ordering of the network, as measured by expectivity, increases with greater connectivity. 


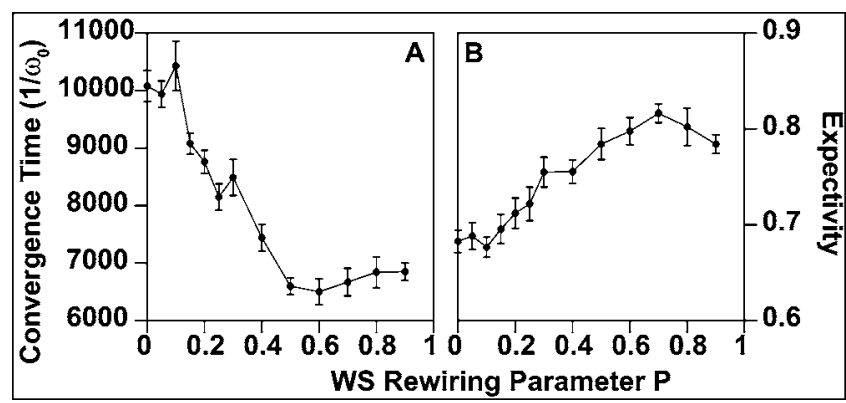

FIG. 4. A 2D regular lattice network of $N=36$ and $r=2$ underwent rewiring as per Watts-Strogatz (Ref. 35) with the end of each coupling rewired to a random oscillator (excluding current connections and self) with probability $P=[0,1.0]$. Larger probabilities results in large amounts of nonlocal connections, which reduce the average distance between oscillators on the network. After a free period of 20000 iterations $\left(1000 \omega_{0}^{-1}\right)$, the network underwent adaptation with speed $\beta_{\omega}=0.0001$. (A) Convergence time of adaptation vs small world rewiring parameter. Larger rewiring probability lead to a shorter time required to completely adapt the oscillators on the network. (B) Expectivity vs small world rewiring parameter. The expectivity reveals that the oscillators time order themselves more effectively as the rewiring parameter is increased, revealing greater communication in the network, and leading to faster adaptation convergence.

network. This is consistent with the results obtained by others showing that the synchrony is more easily obtained for networks with global connectivity. ${ }^{30-32}$

Finally, to test the effectiveness of adaptation in the presence of noise, a noise term $\zeta \varepsilon$ was added to the equation for the $y$ oscillator coordinate, where $\varepsilon$ is a uniformly distributed random value on the interval $[-1,1]$ and $\zeta$ is the noise amplitude. The completeness of the adaptation at $t=20000 \omega_{0}^{-1}$ is measured with the ratio of the final to initial standard deviation of the frequencies of the oscillators. The ratio of 1 indicates that no significant adaptation of oscillator frequencies was achieved. The results (Fig. 5, where $\zeta$ is normalized by the characteristic amplitude of the oscillator $=10$ ) reveal that systems with large values of noise may still undergo a large degree of completion of the adaptation process in the same period of time for which the noiseless network will completely adapt. Furthermore, the adaptation is not completely disrupted until the noise is nearly an order of magnitude higher than the oscillator amplitude, indicating relatively large robustness of the process. Finally, it can be observed that for a range of noise amplitudes $(\zeta=1-3.5)$, networks with moderate and large proportion of long-range connections have more efficient adaptation than networks with only local coupling.

In biological networks the global adaptation may have negative consequences as it may lead to transient indiscriminate synchronization of neurons during epileptic seizure. Formation of spurious connections (axonal sprouting), which lead to changes in network topology was implicated as a possible mechanism leading to epilepsy. ${ }^{33,34}$

\section{B. Network rewiring driven by temporal interdependence between the network elements}

In this set of simulations the network was allowed only to rewire, based on temporal interdependence between the oscillators [Eq. (3)]. The adaptation of oscillator frequencies was not present.

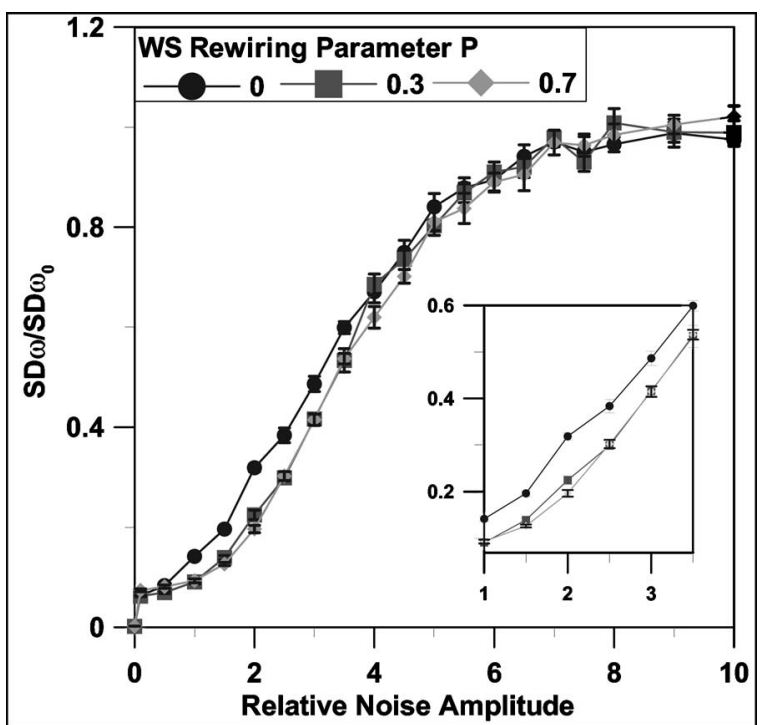

FIG. 5. Standard deviation of frequency (SD $\omega)$ at the end of the simulation, normalized by the initial $\mathrm{SD} \omega_{0}$, vs relative noise amplitude (noise amplitude $\zeta$ normalized by maximal oscillator amplitude). After a period of 20000 iterations $\left(1000 \omega_{0}^{-1}\right)$, adaptation was allowed to occur with speed $\beta_{\omega}$ $=0.00005$, and was allowed to continue for 380000 iterations $\left(19000 \omega_{0}^{-1}\right)$. The average standard deviation of the frequency $\omega$ was measured at the end of each of ten simulations for each value of noise amplitude and small world rewiring parameter $P$. For moderate ranges of noise, larger values of $P$ result in a tighter distribution of frequency, indicating greater progress toward complete adaptation (see inset).

An example of the evolution of such a network is presented in Fig. 6. The initial highly connected network in this example evolves to a sparsely connected one. Note that the clusters form unidirectional connections from the oscillators having higher frequency to the oscillators having a lower one, creating clusters with directed information flow. This is due to the structure of the rewiring function employed for network rewiring. A similar function is thought to be responsible for activity dependent synaptic plasticity in the biological neuronal networks, indicating that such directional clusters may form in the brain.

We have investigated the cluster formation in the presence of noise (Fig. 7). As before, we applied the noise term $\zeta \varepsilon$ to the oscillator $y$ coordinate. The transitivity decreases with the noise amplitude, indicating that increased levels of noise gradually reduce the ability of oscillators to synchro-

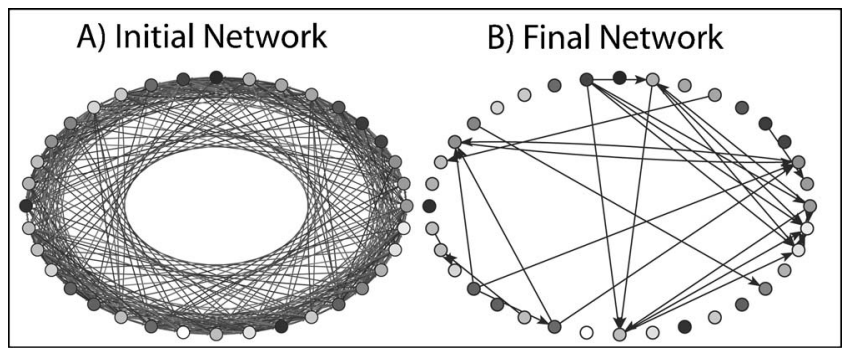

FIG. 6. A network undergoing rewiring $\left(N=36, r=2, \beta_{\alpha}=100\right)$. The node colors represent relative oscillator frequency. During the run time of 20000 $\omega_{0}^{-1}$, the rewiring process pares down most of the couplings in the network, leaving clusters of oscillators with similar frequencies. The coupling is unidirectional, with the faster (darker) oscillators leading to the slower ones. 


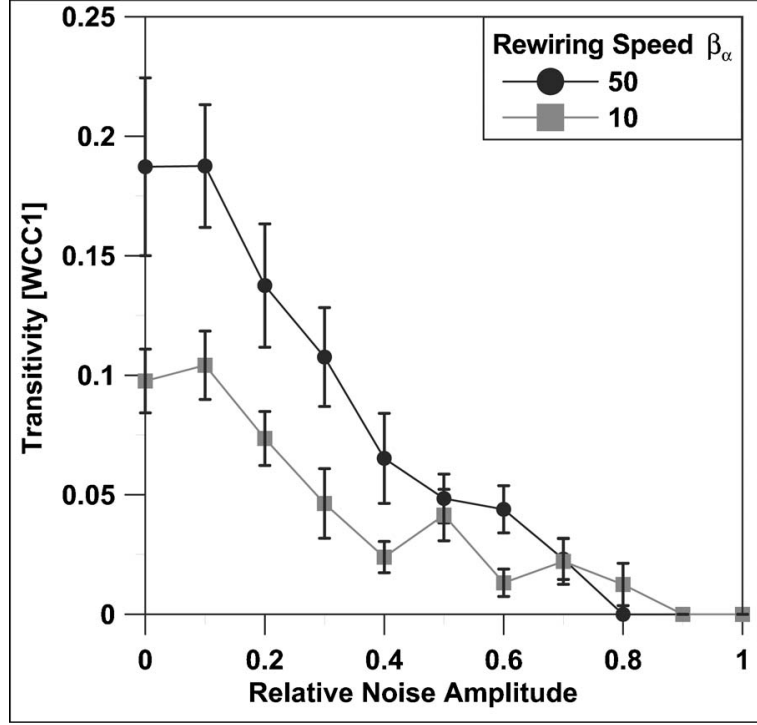

FIG. 7. Average weighted transitivity vs relative noise amplitude (noise amplitude $\zeta$ normalized by characteristic oscillator amplitude). After a period of 20000 iterations $\left(1000 \omega_{0}^{-1}\right)$, the network underwent rewiring with speed $\beta_{\alpha}=\{10,50\}$. Increased noise disrupts the synchrony, and encourages the loss of coupling while discouraging the formation of new couplings. Thus the number of couplings as well as the number of intracluster couplings decreases with noise on the order of a oscillator amplitude.

nize and form highly transitive clusters. However, it takes a noise level of roughly the characteristic amplitude of the oscillators to completely suppress clustering due to rewiring. Still, rewiring is more sensitive to noise than is adaptation, which can withstand more than five times the noise before ceasing to function.

The rewiring function, as described in the introduction [Eq. (3)], is itself a symmetric function, i.e., the slope of the rewiring does not favor strengthening (coalescence) or weakening (fragmentation) of couplings. It is the value of the CEs in relation to the threshold that determines whether the connection forms (the network coalescences) or weakens and gets eliminated (the network fragments). The threshold value, $\mathrm{CE}_{\text {threshold }}$ selects a reasonable level of synchrony required to differentiate between the two processes. Therefore, shifting the threshold will affect not the rewiring itself, but the degree of temporal locking required to form the connection between an oscillator pair. This symmetry in coalescence and fragmentation maybe broken, however, by modifying the rewiring function to be asymmetrical. The rewiring can be then defined to be

$$
\begin{aligned}
& \Delta \alpha_{i j}=\frac{\beta_{\alpha}}{N}\left(C E_{\text {threshold }}-C E_{i j}\right), \\
& \beta_{\alpha}= \begin{cases}\beta_{\alpha f} & \text { if } \mathrm{CE}_{\text {threshold }}<\mathrm{CE}_{i j} \\
\beta_{\alpha c} & \text { if } \mathrm{CE}_{\text {threshold }}>\mathrm{CE}_{i j},\end{cases}
\end{aligned}
$$

where $\beta_{\alpha c}$ is the coalescence constant and $\beta_{\alpha f}$ is the fragmentation constant. The two parameters define the relative speed of coalescence and fragmentation in the network. We studied the selectivity of the cluster formation - defined as the ratio of the local (within cluster) to global (networkwide) standard deviation of oscillator frequencies

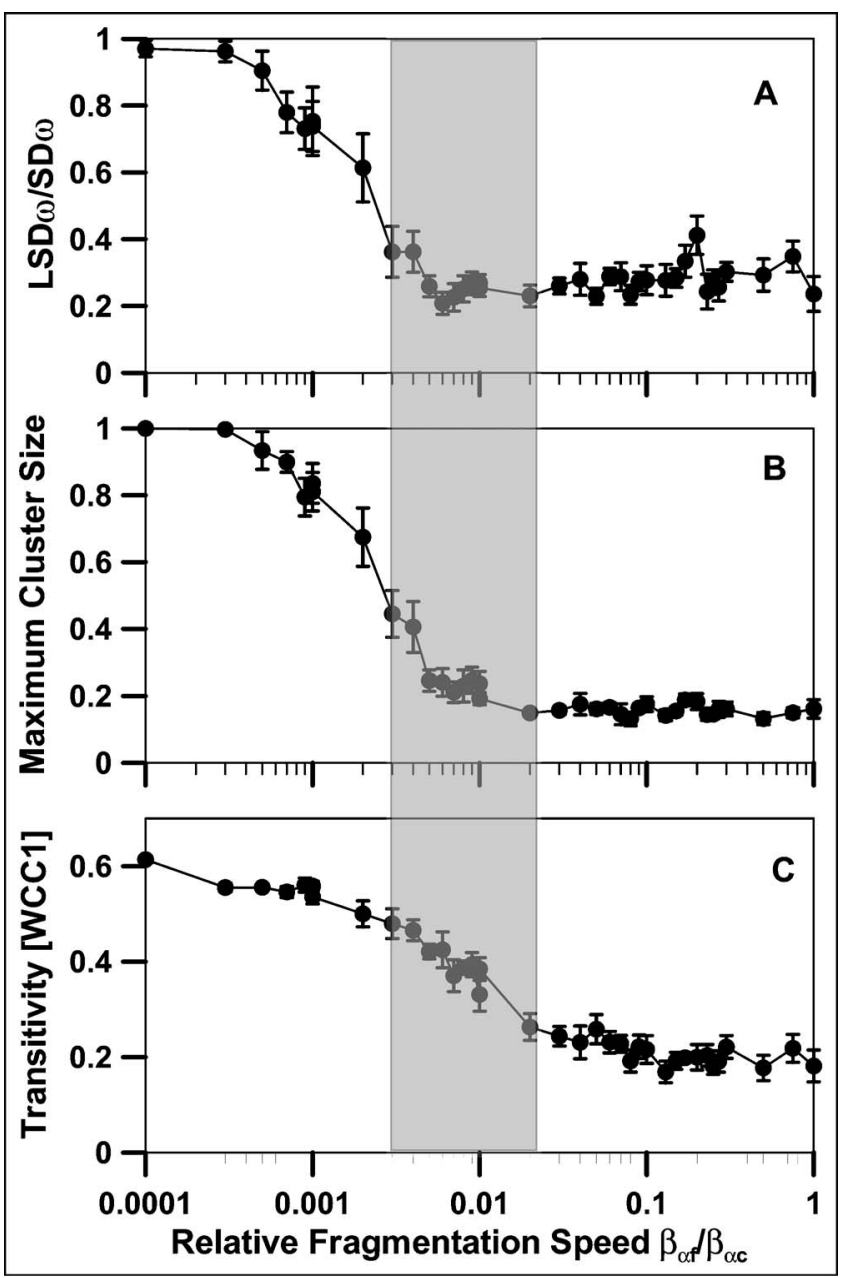

FIG. 8. (A) Relative local standard deviation of frequency, (B) maximum cluster size (normalized by $N$ ), and (C) average transitivity for asymmetric rewiring. After an initialization period of 20000 iterations $\left(1000 \omega_{0}^{-1}\right)$, asymmetric rewiring occurred on a network $(N=36, r=2)$ for a period of 380000 iterations $\left(19000 \omega_{0}^{-1}\right)$. The fragmentation speed was varied, while the coalescence speed was kept constant at $\beta_{\alpha c}=100$. The gray box represents the region in which the selectivity of the clusters is enhanced while relatively high transitivity is maintained.

$\mathrm{LSD} \omega / \mathrm{SD} \omega$; the lower this ratio, the more selective are the clusters - as well as the size of the maximal cluster formed and the transitivity as a function of the ratio of coalescence to fragmentation constant (Fig. 8). For low relative values of $\beta_{\alpha f}$ transitivity and cluster size tend to 1 , but with a loss of oscillator selectivity ( $\mathrm{LSD} \omega / \mathrm{SD} \omega>1)$. This indicates that the there is a large population of connections formed so that one cluster is formed over the whole network; this regime holds no advantage over shifting the CE threshold. For higher values, though, there is a region in which transitivity (WCC1) is strengthened while selectivity $(\mathrm{LSD} \omega / \mathrm{SD} \omega)$ is largely or completely conserved. In this region the maximum cluster size scales roughly with the selectivity, suggesting that the asymmetric rewiring can improve the quality of clusters (in terms of higher transitivity) by adding coupling within clusters rather than adding more oscillators to a cluster. The decreasing maximal cluster size indicates formation of relatively small, highly selective, densely packed clusters in the network. 


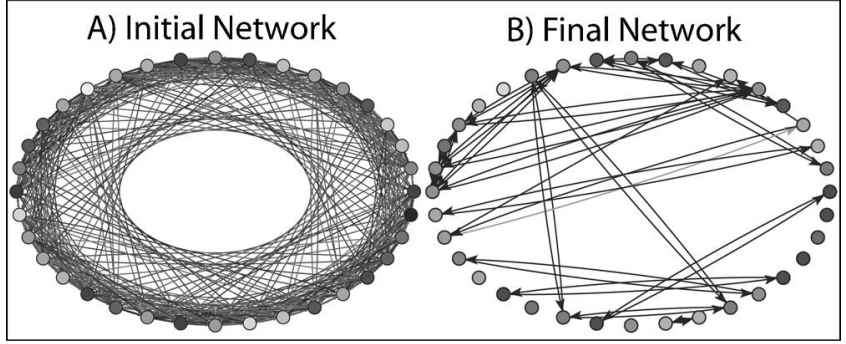

FIG. 9. A network undergoing simultaneous adaptation and symmetric rewiring $\left(N=36, r=2, \beta_{\omega}=0.000316, \beta_{\alpha}=100\right)$. The colors denote relative frequency of the oscillators. After $20000 \omega_{0}^{-1}$, the network is composed of clusters of oscillators with very similar frequencies. Most coupling is bidirectional, indicating the formation of feedback loops and the complete synchronization of oscillators within the cluster.

For the symmetric rewiring function (the ratio $\approx 1$ ) relatively few, small, selective clusters are formed.

\section{Adaptation and rewiring}

In this set of simulations both processes, adaptation and symmetric rewiring $\left(\beta_{\alpha c}=\beta_{\alpha f}=\beta_{\alpha}\right)$, were allowed to take place simultaneously to modify the network structure.

An example of network behavior is shown in Fig. 9. The network forms relatively small clusters from oscillators having the same final frequency. This in turn leads to the formation of feedback loops, or bidirectional connections between the elements of the cluster. Thus contrary to the previous case (rewiring only) the clusters do not have preferred direction of information flow.

We have investigated the resulting network topology for different relative speeds of adaptation and rewiring processes. The limiting cases are suggested from the results above that focus on each process individually. When adaptation is much slower than rewiring, the network first breaks into clusters with similar frequencies, which then are adapted toward a common value for each cluster. When adaptation is much faster than rewiring, the oscillators may adapt to a common frequency and then coalesce into one large cluster. However, adaptation speed can be limited by connectivity and topology of couplings, as demonstrated in the adaptation section. Furthermore, the synchronizability of the network can be limited not only by the distribution of oscillator frequencies, but also by the initial structure of the network itself, particularly its connectivity. Thus, another limiting case for slow rewiring is that the network, though with high adaptation, is still not globally synchronizable state, so that the rewiring processes gradually tear the network apart [see Figs. 10(B) and 10(D)].

We have studied network selectivity, maximal cluster size, transitivity for different relative values of network rewiring, and adaptation for different values of initial connectivity. Figure 10(A) shows the average standard deviation of frequency within clusters normalized by the standard deviation of frequency across all oscillators in the system $(\mathrm{LSD} \omega / \mathrm{SD} \omega)$. The curve obtained for $\beta_{\omega}=0$ shows the selectivity of the rewiring process. There is a tendency for couplings to remain between oscillators with more similar frequencies. In this case the selectivity is around $30 \%$ of the

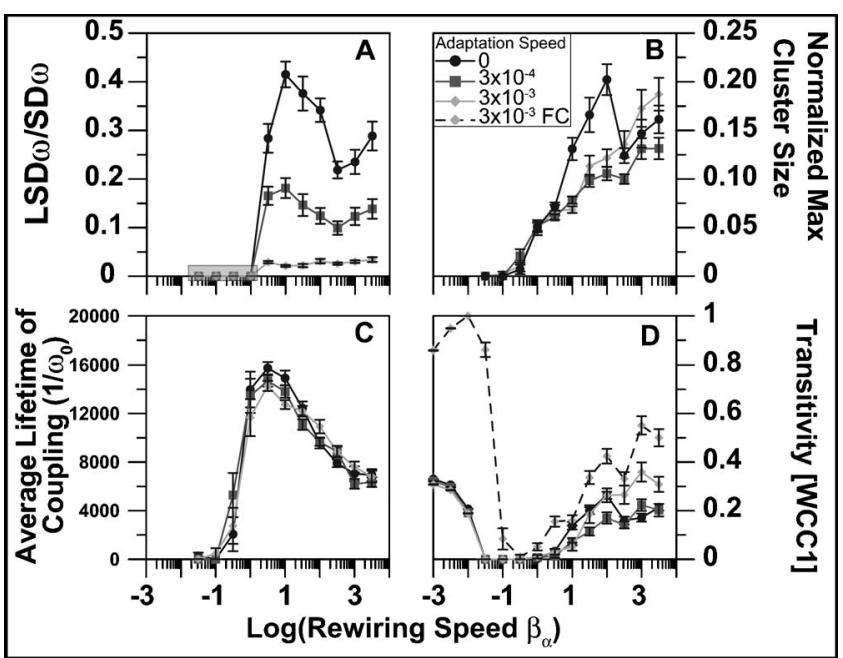

FIG. 10. Network measures vs rewiring speed $\beta_{\alpha}$ for various adaptation speeds $\beta_{\omega}$. A network $(N=36, r=2)$ was integrated for 20000 iterations $\left(1000 \omega_{0}^{-1}\right)$ before adaptation and rewiring were allowed for 380000 iterations $\left(19000 \omega_{0}^{-1}\right)$. Each data point is the result of ten simulations. (A) Standard deviation of $\omega_{i}$ within clusters (normalized by network-wide standard deviation of $\left.\omega_{i}\right) \mathrm{LSD} \omega / \mathrm{SD} \omega$. For zero adaptation $\left(\beta_{\omega}=0\right), \mathrm{LSD} \omega / \mathrm{SD} \omega$ shows the selectivity of the rewiring processes. Rewiring selects oscillators with roughly $30 \%$ of the available range of frequencies when clustering forms. With adaptation, established clusters can adapt to become more similar. For parameters which resulted in no clusters, LSD $\omega / \mathrm{SD} \omega$ is undefined; these points are marked with a gray rectangular overlay. (B) Maximum cluster size (normalized by the size of the network). Greater rewiring speed results in larger maximum cluster size. (C) Average lifetime of couplings. For very low values of rewiring speed, the network dissociates, with all couplings vanishing. Rarely, new couplings will be generated for short periods of time. For moderate values of rewiring speed, stable couplings are generated between compatible oscillators. For large values of $\beta_{\alpha}$, the network again begins to dissociate. The reduced coupling lifetime is due to both the tendency for couplings to be deleted and for new, unstable couplings to be generated. (D) Average weighted transitivity. There is a tendency towards increased transitivity with faster rewiring, until it plateaus. Larger values of adaptation speed allow for continued increase in transitivity after a plateau occurs for smaller adaptation speeds. The dashed line represents initially full connectivity (coupling between all pairs in each direction, but with random coupling strength). It is shown that for slow rewiring ( $\beta_{\alpha}$ $=0.03$ ) a completely transitive cluster emerges, with all couplings at full strength. For values of rewiring slower than this, run time was not sufficient to completely aggregate the cluster.

range of available frequencies for rewiring such that $\beta_{\alpha}>1$. Increased $\beta_{\omega}$ adapts clusters that have formed to become more uniform, resulting in a local standard deviation of frequency near zero. For slower rewiring, the network breaks up due to the lack of time ordering during the gradual decline of the coupling. This lack of clusters leads to an undefined local standard deviation within the cluster (gray region).

The relation between maximal cluster size and the relative speed of adaptation and network rewiring remains unclear [Fig. 10(B)]. The increase in the rewiring speed creates larger and more transitive clusters [Fig. 10(D)], while faster adaptation does not appear to clearly affect the size of the clusters [Fig. 10(B)]. However, adaptation is strengthening transitivity amongst clusters formed with fast rewiring [Fig. 10(D)]. This is due to the fact that the adapted clusters form bidirectional connectivity.

Lastly, we have studied the lifetime of the connections between the oscillators as a function of rewiring speed and adaptation speed [Fig. 10(C)]. The decrease in average life- 
time of couplings with an increase in rewiring speed is due to both the continued creation of new couplings further into the simulation and an increased tendency to destroy couplings when synchrony is interrupted.

The mechanism for the formation of clusters appears to rely upon two features. First, couplings are pared from the initial lattice, leaving couplings between synchronized oscillators, usually with similar frequencies. These small clusters then "capture" other oscillators that trajectories happen to briefly coincide with to one of the members of the cluster. A coupling forms, and the coupled trajectories begin to synchronize. At the same time, trajectories of other members of the cluster will begin to emulate or be emulated by the new member, causing synchronization over the whole cluster and causing new couplings to be formed, leading to a highly transitive cluster.

\section{CONCLUSIONS}

We have studied network reorganization driven by temporal interdependencies between elements in the system of coupled, initially nonidentical Rössler oscillators. The temporal interdependencies in the network are monitored by an adaptive, asymmetric measure that monitors changes in phase/lag synchrony between its oscillator pairs. We have studied two basic mechanisms of network reorganization: adaptation of frequencies of individual oscillators and network rewiring based on asymmetries in temporal interdependencies of their dynamics. Both of those mechanisms led separate processes during network reorganization. Adaptation led to formation of large clusters composed of identical oscillators, whereas rewiring alone led to formation of smaller clusters having directed information flow. Both processes together led to the formation of adapted clusters with bidirectional couplings producing feedback loops between its elements. Additionally we have found the existence of an optimal ratio of relative coalescence and fragmentation speeds during asymmetric rewiring during which highly selective large clusters are formed. This prediction should now be investigated experimentally during LTP and LTD processes in the brain. On the other hand, for a range of relative speeds of adaptation and rewiring the initial network connections are effectively destroyed leading to annihilation of the network.

\section{ACKNOWLEDGMENTS}

This research was done with the support of National Institutes of Health (1 R21 EB003583) (M.Z.) and NIH Molecular Biology Training Grant No. 2T32GM08270-17 (J.W.). The authors would like to thank the Center for the Study of Complex Systems at the University of Michigan for the use of their computer systems for this investigation.

${ }^{1}$ J. Liu, J. Wu, and Z. Yang, Physica A 341, 273 (2004).

${ }^{2}$ R. M. May and A. L. Lloyd, Phys. Rev. E 64, 066112 (2001).

${ }^{3}$ M. Newman, Phys. Rev. E 64, 016313 (2001a).

${ }^{4}$ M. Newman, Phys. Rev. E 64, 016313 (2001b).

${ }^{5}$ J. Torres, M. Munoz, J. Marro, and P. Garrido, Neurocomputing 58, 229 (2005)

${ }^{6}$ G. Bianconi, M. Marsili, and F. Vega-Redondo, Physica A 346, 116 (2005).

${ }^{7}$ A. Barabàsi and R. Albert, Science 286, 509 (1999).

${ }^{8}$ A. Venaille, P. Varona, and M. Rabinovich, Phys. Rev. E 71, 061909 (2005).

${ }^{9}$ C. Assisi, V. Jirsa, and S. Kelso, Phys. Rev. Lett. 94, 018106 (2005).

${ }^{10}$ R. Vilela Mendes, Int. J. Bifurcation Chaos Appl. Sci. Eng. 15, 1185 (2005).

${ }^{11}$ M. Argollo de Menezes and A. Barabàsi, Phys. Rev. Lett. 92, 028701 (2004).

${ }^{12}$ A. Luthi and D. McCormick, Neuron 21, 9 (1998).

${ }^{13}$ L. Abbott and S. B. Nelson, Nat. Neurosci. 3, 1178 (2000).

${ }^{14}$ H. Tao, L. Zhang, G. Bi, and M. Poo, J. Neurosci. 20, 3233 (2000).

${ }^{15}$ R. Fitzsimonds, H. Song, and M. Poo, Nature (London) 388, 439 (1997).

${ }^{16}$ C. Zhou and C.-H. Lai, Phys. Rev. E 59, 6629 (1999).

${ }^{17}$ T.-L. Liao and S.-H. Tsai, Chaos, Chaos, Solitons Fractals 11, 1387 (2000).

${ }^{18}$ A. d'Anjou, C. Sarasola, F. Torrealdea, R. Orduna, and M. Grana, Phys. Rev. E 63, 046213 (2001).

${ }^{19}$ M. Zochowski and R. Dzakpasu, J. Phys. A 37, 3823 (2004).

${ }^{20}$ R. Dzakpasu and M. Zochowski, Chaos 14, 583 (2004).

${ }^{21}$ M. Pecora and T. Carroll, Phys. Rev. Lett. 64, 821 (1990).

${ }^{22}$ L. Kocarev and U. Parlitz, Phys. Rev. Lett. 77, 2206 (1996).

${ }^{23}$ L. Kocarev and U. Parlitz, Phys. Rev. Lett. 76, 1816 (1996).

${ }^{24}$ N. Rulkov, M. Suschik, L. Tsimring, and D. Abarbanel, Phys. Rev. E 51, 980 (1995).

${ }^{25}$ W. Gerstner, Phys. Rev. Lett. 76, 1755 (1996).

${ }^{26}$ U. Parlitz, L. Junge, W. Lauterborn, and L. Kocarev, Phys. Rev. E 54, 2115 (1996).

${ }^{27}$ U. Ernst, K. Pawelzik, and T. Geisel, Phys. Rev. Lett. 74, 1570 (1995).

${ }^{28}$ M. Rosenblum, A. Pikovsky, and J. Kurths, Phys. Rev. Lett. 78, 4193 (1997).

${ }^{29}$ D. van den Berg and C. van Leeuwen, Europhys. Lett. 65, 459 (2004).

${ }^{30}$ M. Barahona and L. M. Pecora, Phys. Rev. Lett. 89, 054101 (2002).

${ }^{31}$ H. Hong, M. Choi, and B. Kim, Phys. Rev. E 65, 026139 (2002).

${ }^{32}$ T. Nishikawa, A. Motter, Y.-C. Lai, and F. Hoppensteadt, Phys. Rev. Lett. 91, 014101 (2003).

${ }^{33}$ T. Netoff, R. Clewley, S. Arno, T. Keck, and J. White, J. Neurosci. 24 8075 (2004)

${ }^{34}$ B. Percha, R. Dzakpasu, M. Zochowski, and J. Parent, Phys. Rev. E 72, 031909 (2005).

${ }^{35}$ D. Watts and S. Strogatz, Nature (London) 393, 440 (1998). 